\title{
Influence du réseau poreux sur l'altération par le gel des massifs calcaires fracturés
}

M. BOST

A. POUYA

S. GUÉDON

Laboratoire Central des Ponts et Chaussées 58 , boulevard Lefebvre 75015 Paris bost@lcpc.fr
En milieu montagneux, un des processus d'altération prépondérants des massifs fracturés est la propagation du réseau de fissures existant par gel-dégel. Afin

d'évaluer la cinétique de ce type d'altération, la génération de contrainte le long d'une fissure artificielle au cours du gel a été étudiée en laboratoire. Les résultats indiquent que le type de réseau poreux de la roche a une incidence directe sur la contrainte générée par le gel dans une fissure. La valeur maximale de la contrainte est limitée par la perméabilité de la roche.

Mots-clés : calcaire, propagation de la fissuration, cycles de gel-dégel, contrainte, réseau poreux, perméabilité.

\section{Influence of porous network on cracked rock mass weathering by freezing-thawing}

In mountainous area one of the dominant rock weathering processes is crack propagation due to freezing-thawing cycles. This mechanism within a cracked rock matrix was reproduced in laboratory with notched specimens to study its kinetic. Experimental results show that the type of porous network of the rock has a direct incidence on the stress generated by freezing in a crack. Maximum value of the stress in crack is bounded by rock permeability.

Key words: limestone, crack propagation, freeze-thaw cycles, stress, porous network, permeability.
} 


\section{1}

\section{Introduction}

Les chutes de pierres posent des problèmes d'aménagement et de gestion du territoire dans les régions montagneuses (Fig. 1 ; PIARC G2, 1999 ; Interreg IIC, 2001). Afin de pouvoir dimensionner les infrastructures exposées et/ou décider de mettre en place des dispositifs de protection adaptés, l'occurrence temporelle du phénomène dans les zones potentiellement instables doit être déterminée. Or si le degré de stabilité d'un versant rocheux peut être estimé, son évolution au cours du temps est mal connue.

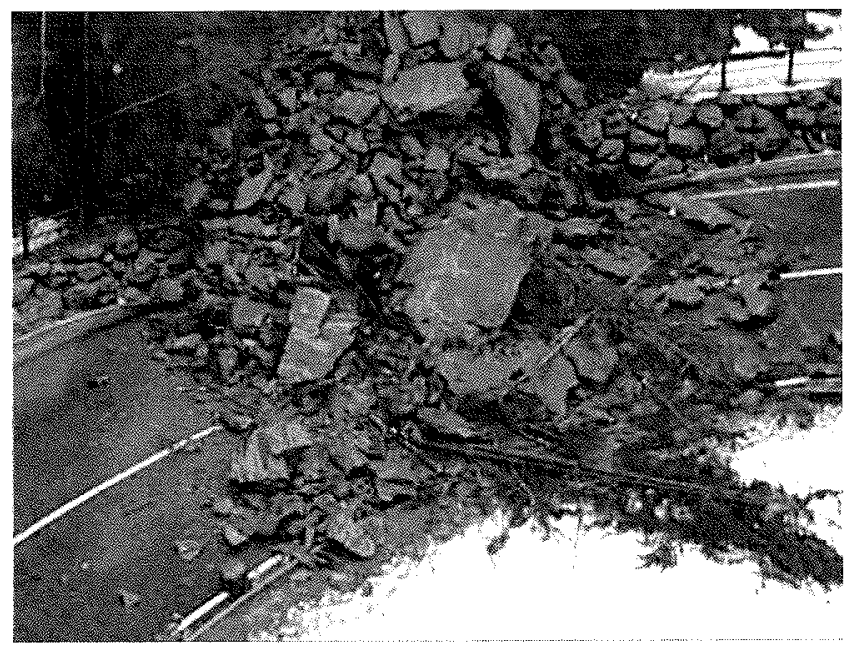

FIG 1 Éboulement sur la route d'accès au MontBlanc (mars 2007, image AFP).

Rockfall on Mont Blanc mountain road.

En milieu montagneux, on peut considérer qu'un des processus d'altération prépondérants des massifs fracturés est la propagation du réseau de fissures existant par gel-dégel (Matsuoka, 2001a ; Frayssines, 2005). Si le mécanisme de gel-dégel au sein d'une matrice rocheuse ou microgélivation a été largement étudié, en laboratoire en particulier, le phénomène reste encore incompris quand, au réseau de pores, s'ajoute un réseau de fissures (macrogélivation) (Matsuoka, 2001b). Le nombre limité d'essais en laboratoire et la complexité des observations sur le terrain ont rendu difficile jusqu'ici l'établissement d'un modèle du processus de macrogélivation dans les roches.

L'objectif des recherches menées au LCPC a été d'améliorer l'évaluation de la sensibilité aux alternances de gel et de dégel, des falaises rocheuses, en termes de chutes de blocs rocheux. Il s'avérait nécessaire d'évaluer non seulement la cinétique d'altération par gel-dégel des massifs rocheux fracturés, mais également les facteurs dont cette dernière dépend. Pour cela, un dispositif expérimental a été mis au point afin d'étudier, en conditions de gel, la génération de contraintes le long d'une fissure artificielle dans un cube de roche. Nous nous sommes limités, dans le cadre de cette recherche, aux roches calcaires : la forte teneur en calcite des calcaires choisis a permis de s'affranchir, dans un premier temps, de la composition minéralogique de la roche.

\section{Reproduction du gel de l'eau dans une entaille d'un cube de calcaire}

\section{8}

\section{Principe}

Pour caractériser la cinétique de propagation d'un réseau de fracturation dans un massif sous l'action du gel, il faut connaître les sollicitations auxquelles sont soumises les fissures existantes sous l'effet du gel. Notre démarche expérimentale a consisté à reproduire le phénomène d'avancement de fissure par action du gel à l'extrémité d'une unique entaille que nous avons façonnée dans un bloc de calcaire (Bost, 2008). Le mot " entaille » définit dans ces travaux, toute fissure artificiellement façonnée pour les besoins de l'expérimentation.

\section{8}

\section{Dispositif expérimental}

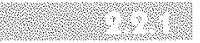 \\ Géométrie de l'éprouvette}

Pour reproduire le cas simple d'une fissure parallèle à la paroi verticale d'un affleurement rocheux, chaque bloc de calcaire a été taillé sous forme cubique puis entaillé par l'intermédiaire d'un trait de scie (Fig. 2). Les extrémités latérales ont été étanchées par un produit souple et un joint torique chargé de reprendre la contrainte (Fig. 3). L'entaille étant remplie d'eau, le cube a été soumis à des cycles successifs de gel-dégel jusqu'à sa rupture. Au cours des cycles, la température et la pression en différents points de l'entaille ont été suivies (Fig. 3).

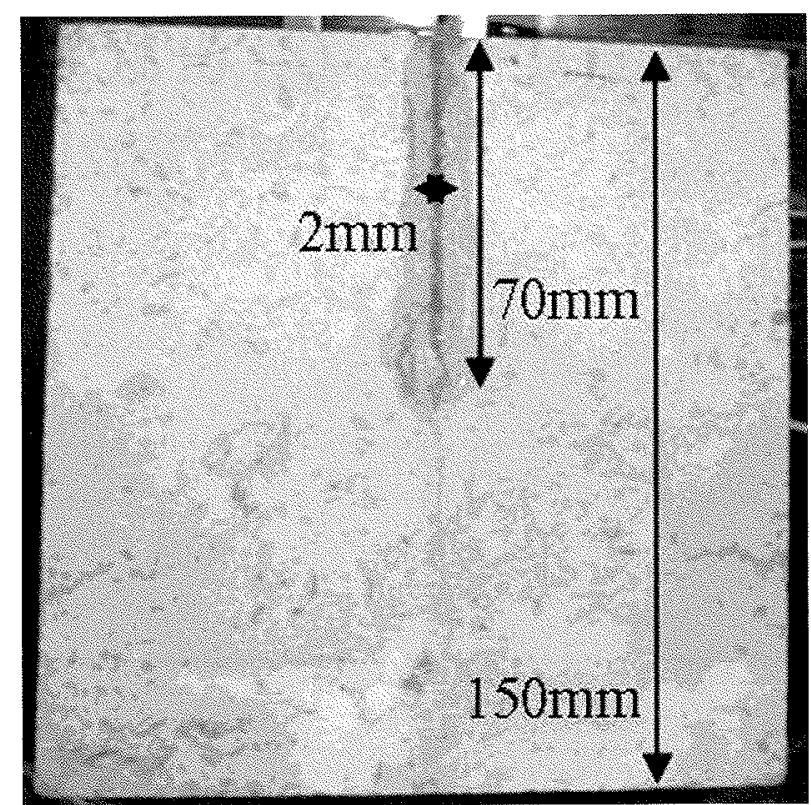

FiG. 2 Dimensions des éprouvettes de calcaires entaillées.

Size of notched limestone specimens. 


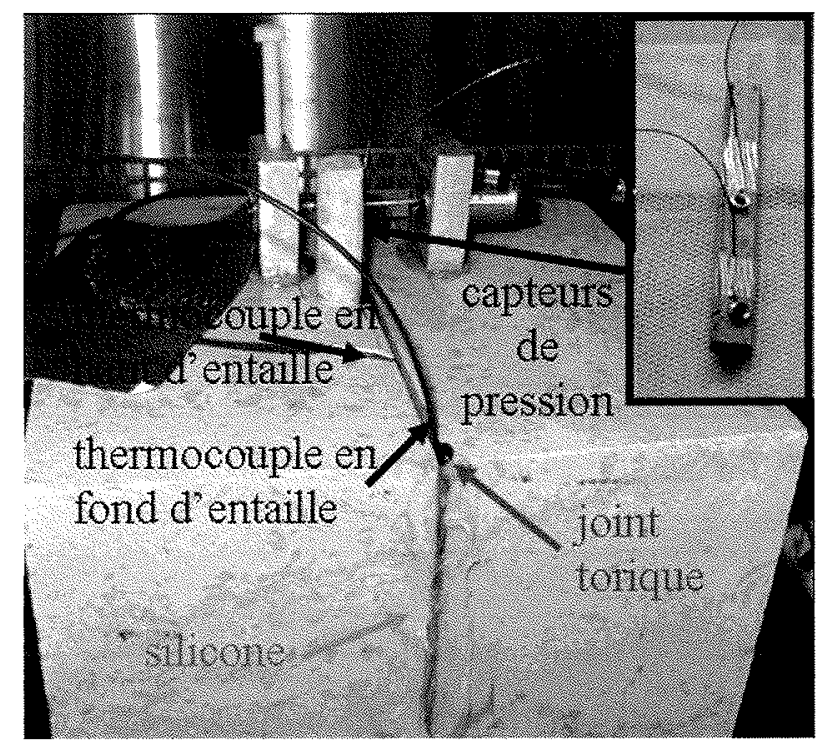

16. 3 Cube de calcaire entaillé et instrumenté. Notched limestone cube with instruments.

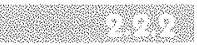

\section{Reproduction des cycles de gel-dégel}

Afin de pouvoir extrapoler les résultats ainsi obtenus en laboratoire au massif rocheux en situation réelle, il était certes important d'imposer des conditions de variations de températures réalistes mais la lenteur du processus demandait également de choisir des conditions relativement sévères. Nous avons donc choisi d'imposer sur toutes les faces du cube un gel relativement doux en mettant l'enceinte frigorifique à $-5^{\circ} \mathrm{C}$ mais sans mise en température graduelle : l'étuve passe directement de la température de gel à la température de dégel $\left(20^{\circ} \mathrm{C}\right)$ sans palier intermédiaire et vice versa.

Les calcaires étudiés ayant naturellement un degré de saturation en eau élevé, nous avons décidé de tester les cubes après saturation à l'eau sous vide. Pour maintenir ce degré de saturation tout au long des cycles et éviter les pertes par évaporation, extrusion et autre migration d'eau (Tourenq, 1970), les éprouvettes de calcaire ont été placées dans un récipient contenant une tranche d'eau de l'ordre du centimètre.

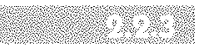

\section{Instrumentation}

La température de l'air dans l'étuve ne permet pas de rendre compte des variations de température dans la roche (Hall et al., 2001) et en particulier dans l'entaille. La température a été suivie au cours des cycles, non seulement dans l'enceinte mais également en haut de l'entaille et en fond d'entaille par des thermocouples (Fig. 3).
Même si, lors du changement de phase, l'eau passe de l'état liquide à l'état solide, nous avons fait le choix de mesurer la contrainte le long de l'entaille par des capteurs de pression. Afin d'avoir une mesure de la contrainte exercée par le gel sur les parois de l'entaille, nous avons usiné une encoche supplémentaire au milieu du cube le long d'une paroi de manière à ce que, une fois collés sur un support, les capteurs de pression aient leur surface dans l'alignement de cette paroi de l'entaille (Fig. 3). Selon les éprouvettes, deux ou trois capteurs de pression ont été ainsi positionnés à différentes profondeurs.

\section{3}

\section{Choix des calcaires d'étude}

Nous avons choisi un Larrys comme calcaire de référence car s'il présente une matrice résistante du point de vue de la microgélivation, il renferme naturellement de nombreuses discontinuités qui représentent autant de zones de faiblesse pour les processus de macrogélivation.

C'est un calcaire oolithique. Sa porosité inférieure à $5 \%$ le situe dans la moyenne basse des calcaires. Son rayon moyen d'accès aux pores ( $R$, Tableau $I)$ est relativement faible $(75 \mathrm{~nm})$. Comparativement à ce calcaire, nous en avons sélectionné deux autres, également oolithiques mais à la structure poreuse différente (Tableau I) : la Pierre de Lens de porosité assez élevée $(14 \%)$ mais au rayon moyen d'accès aux pores élevé $(600 \mathrm{~nm})$ et le calcaire de Chamesson de forte porosité $(17,5 \%)$ avec un rayon moyen d'accès aux pores intermédiaire (284 nm).

\section{3}

\section{Résultats et analyses}

\section{3.}

\section{Suivi de la propagation de l'entaille en fissure sous l'action du gel}

Pour les cubes de Chamesson et de Larrys, respectivement un et deux cycles de gel-dégel seulement ont été nécessaires pour qu'une fissure soit visible à l'ceil nu dans le prolongement de l'entaille initiale (Fig. 4). Le cube de Pierre de Lens n'a commencé à s'altérer qu'au bout de cinq cycles.

La sensibilité au processus de macrogélivation diffère donc selon le calcaire. Or les conditions de gel et la géométrie, en particulier des entailles, sont les mêmes pour les trois cubes. Par conséquent, cette différence est intrinsèquement liée aux caractéristiques de la roche.

1. Arume Caractéristiques des calcaires de l'étude (mesures faites en laboratoire selon les normes en vigueur). Limestones properties for this experiment (measurements done in laboratory according to French standards).

\begin{tabular}{|c|c|c|c|c|}
\hline Calcarre & Porroviter (1) & If (hrm) & Ex $(C P)$ & Rt $=(M P r)$ \\
\hline Chamesson & 17,5 & 284 & 34,6 & 6,9 \\
\hline Larrys & 4,3 & 75 & 64,9 & 11 \\
\hline Pierre de Lens & 14 & 600 & 32,2 & 8,05 \\
\hline
\end{tabular}

* $\mathrm{E}=$ module $\mathrm{d}^{\prime}$ Young ; ** Rt = résistance en traction-essai brésilien. 

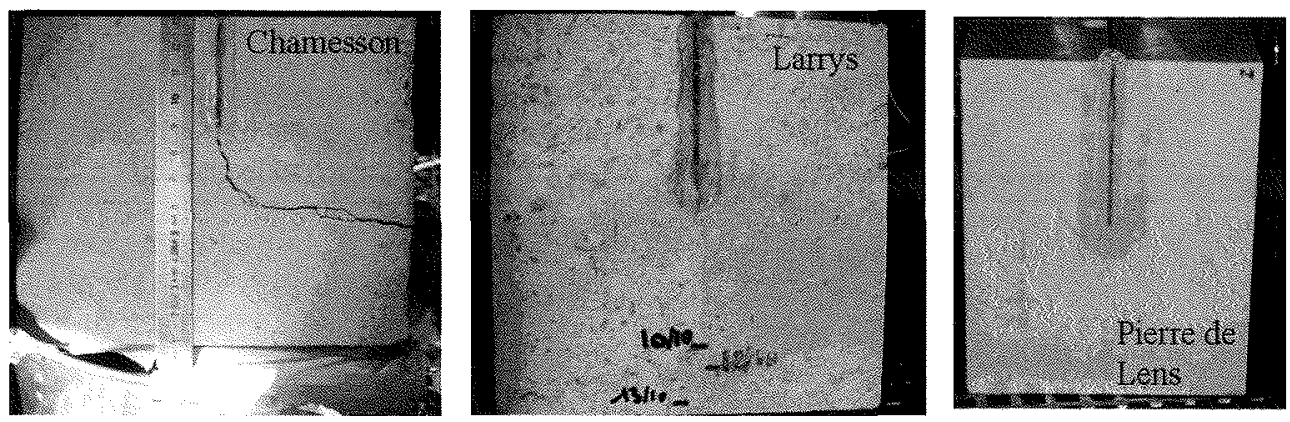

FG. 4. Prolongement de l'entaille en fissure suite aux cycles de gel-dégel, pour les trois calcaires testés.

Crack propagation as a result of freeze-thaw cycles for three tested limestones.

Les trois calcaires testés étant quasiment purs en calcite, leurs caractéristiques thermiques (conductivité thermique, capacité calorifique, coefficient de diffusivité thermique) ne varient que par la valeur de la porosité et l'état de saturation. Or la porosité varie suffisamment peu entre les trois calcaires (Tableau I : de 5 à 17,5\%) pour que l'ordre de grandeur des caractéristiques thermiques diffère (Tableau II). D'autre part, la classification des calcaires selon leur sensibilité au gel dans une entaille ne correspond pas à celle établie selon la résistance à la traction (Tableau I) : le Larrys est moins résistant à la propagation de la fissuration sous l'action du gel que la Pierre de Lens malgré sa résistance à la traction plus importante. La propagation de la fissuration par le gel dans une roche ne peut s'expliquer par la seule augmentation volumique lors du changement de phase de l'eau. La variation du comportement lors du gel entre ces trois calcaires ne peut donc s'expliquer que par des processus complémentaires faisant intervenir la microstructure, propre à chacune des roches testées.

\section{2.}

\section{Suivi de la variation de température en fond d'entaille}

La figure 5 représente la superposition de la variation de la température en fond d'entaille pour les trois calcaires testés au cours d'un cycle de gel-dégel. La durée des phases a été adaptée pour chacun des calcaires en fonction de leur équilibre thermique. La phase de dégel se repère par l'augmentation de la température.

Les variations de la température, que ce soit avant ou après le changement de phase, sont quasiment similaires pour les trois calcaires (Fig. 5). Ainsi la comparaison de la variation de température en fond d'entaille entre les différents calcaires confirme que leurs comportements thermiques sont proches, tant qu'on reste suffisamment éloigné du changement de phase.

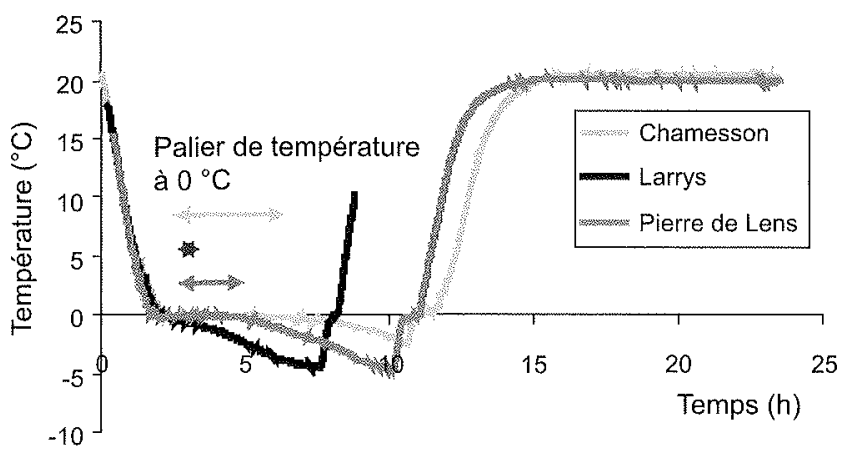

fic. 5 Variation de la température $\left({ }^{\circ} \mathrm{C}\right)$ en fond d'entaille de chacun des trois cubes calcaires testés au cours d'un cycle de gel-dégel. Temperature evolution $\left({ }^{\circ} \mathrm{C}\right)$ at the bottom of the notch for the three tested limestones during a freeze-thaw cycle.

Le changement de phase d'un corps pur à pression constante s'effectue à température constante. Pour le passage de l'état liquide à l'état solide de l'eau pure à la pression atmosphérique, cette température est de $0^{\circ} \mathrm{C}$. Un palier de température à environ $0^{\circ} \mathrm{C}$ est observé en fond d'entaille pour chacun des calcaires (Fig. 5). Quel que soit le calcaire testé, une partie de l'eau contenue dans les éprouvettes testées (réseau poreux et/ou entaille) gèle donc quasiment sans surfusion à la température classique de changement de phase de l'eau liquide. Or la durée de ce palier, différente selon le calcaire, s'avère fortement corrélée à la porosité de la roche (Fig. 6). La durée du palier de gel en système ouvert (expansion volumique non restreinte) est en fait fonction de la quantité d'eau liquide à geler. Or plus le calcaire est poreux, plus la quantité d'eau susceptible de geler est importante. Cette relation entre une caractéristique propre de la roche, la porosité, et la variation de la température de l'eau dans l'entaille semble indiquer que la température dans l'entaille est imposée par celle dans la roche lors du gel de l'eau qu'elle contient.

rabuku II Caractéristiques thermiques des calcaires testés.

Thermic properties of tested limestones.

\begin{tabular}{|c|c|c|c|}
\hline$(2)+1(2)+x)$ & 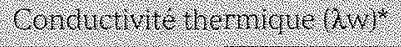 & 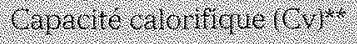 & 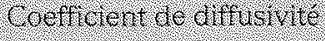 \\
\hline Unité & W.m $\mathrm{m}^{-1} \cdot{ }^{\circ} \mathrm{C}^{-1}$ & $\mathrm{~J} \cdot \mathrm{m}^{-3} \cdot{ }^{\circ} \mathrm{C} \cdot 1$ & $m^{2} \cdot s^{-1}$ \\
\hline Chamesson & 3,0 & $1,13.10^{4}$ & $2,65.10^{\cdots 4}$ \\
\hline Larrys & 3,6 & $1,47.10^{4}$ & $2,45.10^{-4}$ \\
\hline Pierre de Lens & 3,1 & $1,19.10^{4}$ & $2,60.10^{-4}$ \\
\hline
\end{tabular}

* Selon la formule de Bousquié (1979) : $\lambda w=3,8(1-1,25 n)$.

** Selon la formule de Caicedo Hormaza (1991): $\mathrm{Cv}_{\text {calcaire }}=\mathrm{Cv}_{\text {calcite }} \times(1-\mathrm{n})+\mathrm{C}_{\text {veau }} \times \mathrm{n}$ où $\mathrm{n}$ est la porosité de la roche. 


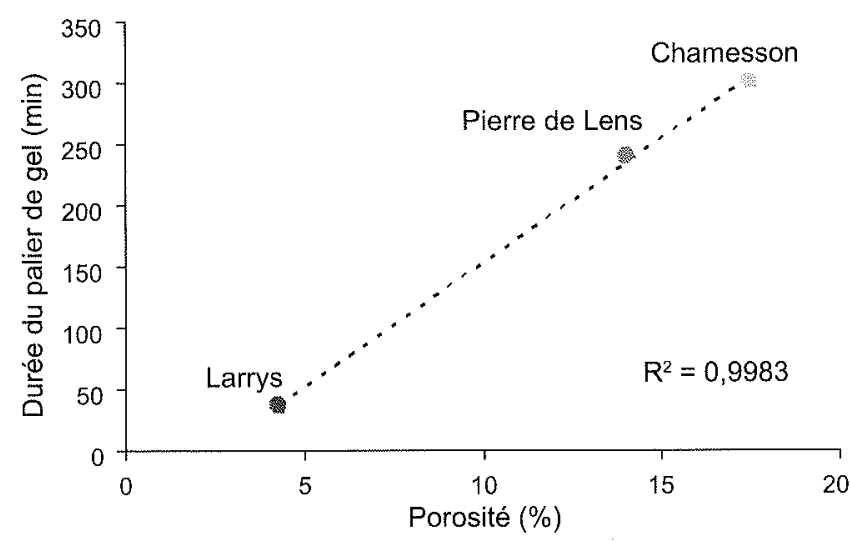

न16. 6 Durée du palier de gel à $0{ }^{\circ} \mathrm{C}$ en fonction de la porosité du calcaire testé.

Duration of « zero curtain effect » period versus tested limestone porosity.
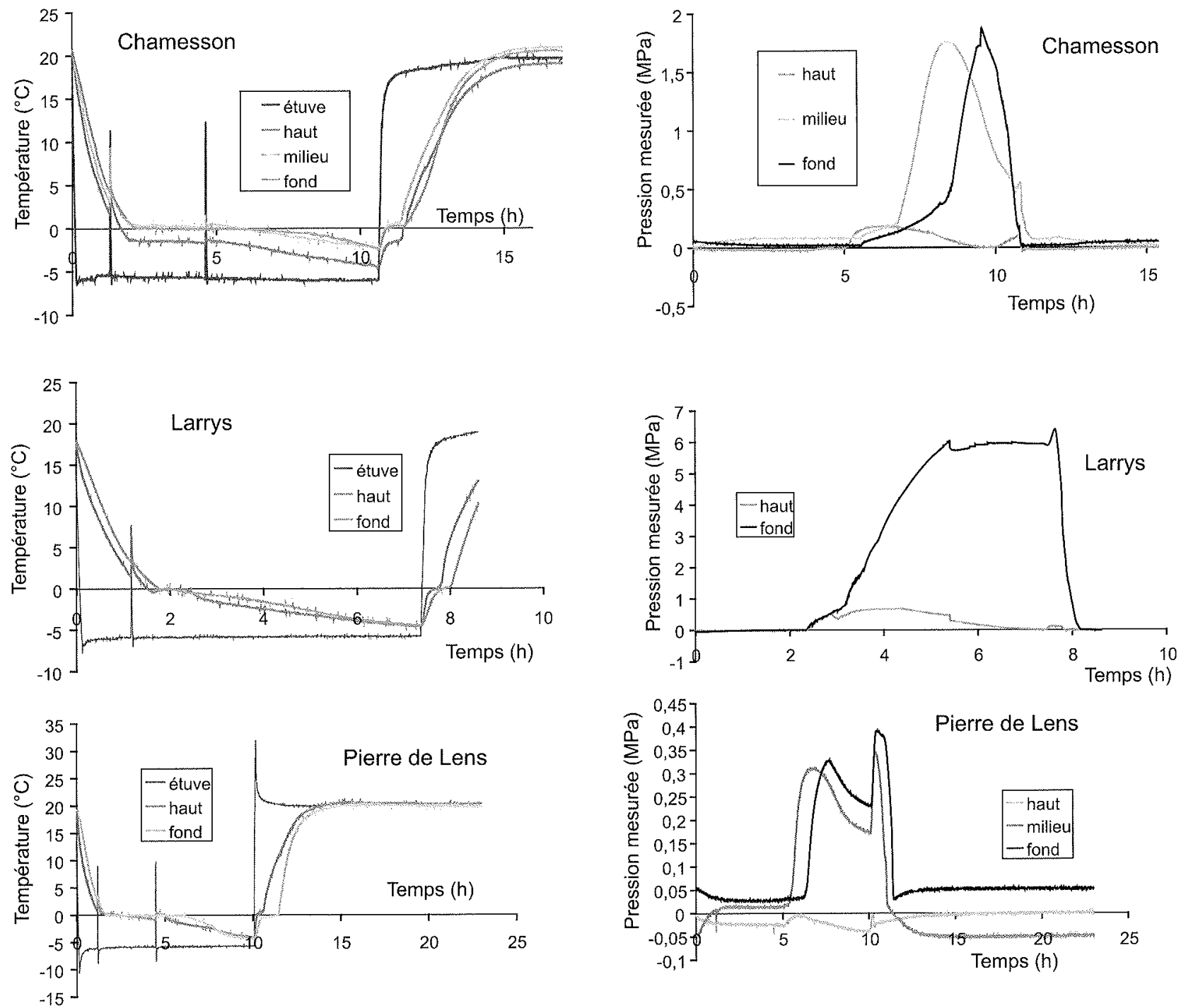

fic. 7 Allure de la température et de la contrainte (MPa) mesurées en différentes profondeurs de l'entaille au cours d'un cycle de gel-dégel pour les trois calcaires.

Evolution of temperature and stress value $(\mathrm{MPa})$ at different depths during a freeze-thaw cycle for the three tested limestones. 
l'eau encore liquide sous-jacente. Puis, quelle que soit la profondeur, la pression augmente jusqu'à une valeur maximale qui est plus importante avec la profondeur dans l'entaille (Fig. 7).

A l'inverse, dans le cas de la Pierre de Lens (Fig. 7), caractérisée par un réseau poreux à gros pores (Tableau I), le palier de température à $0^{\circ} \mathrm{C}$ semble être plus long en profondeur qu'en haut de l'entaille. Il semble que la grande quantité d'eau dans le réseau poreux susceptible de geler à $0^{\circ} \mathrm{C}$ pour cette roche se traduit par une propagation plus lente et donc visible du front de gel au cour de l'échantillon par rapport au cas du Larrys. Par ailleurs, les valeurs de la pression au milieu et en fond d'entaille augmentent en haut de l'entaille d'abord faiblement dès la fin du palier à $0^{\circ} \mathrm{C}$, puis plus fortement successivement dans le temps avec la profondeur. La lente propagation du front de gel et la forte perméabilité de la roche semblent laisser le temps, en fond d'entaille, à l'eau encore liquide de s'échapper dans la roche et justifier ainsi l'augmentation initiale faible de la pression en fond d'entaille. La forte augmentation de pression, qui suit dans le temps au cours du gel, pourrait se justifier par l'arrivée du front de glace dans l'entaille au droit de la zone de mesure. Enfin, seules des migrations d'eau s'opérant par les parois encore non complètement obstruées par la glace peuvent justifier une pression non hydrostatique au sein de l'eau encore liquide dans l'entaille.

Chamesson a un comportement intermédiaire (Fig. 7) : le palier de température en fond d'entaille est un peu plus long que ceux en haut et en milieu d'entaille et la pression augmente de manière quasiment hydrostatique en milieu et en fond d'entaille avec un peu de retard par rapport au haut de l'entaille. Certes ce calcaire est le plus poreux des trois testés (Tableau I) mais son réseau poreux a de moins gros pores que la Pierre de Lens si bien que la propagation du front de gel est relativement rapide et permet, après un certain temps, le confinement de l'eau encore liquide en fond d'entaille. De la même manière que pour la Pierre de Lens, le passage du front de glace dans l'entaille au droit de la zone de mesure pourrait justifier la brusque augmentation de pression que ce soit en milieu ou en fond d'entaille.

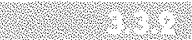 \\ Contrainte maximale au cours d'un cycle de gel-dégel à différentes profondeurs le long de l'entaille}

On peut noter que la valeur et l'allure de la contrainte maximale le long de l'entaille varient selon le cube de calcaire (Fig. 8). La contrainte développée dans

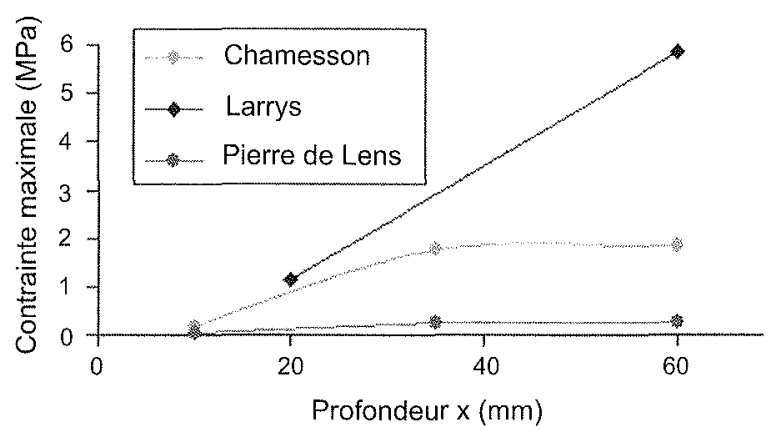

FG, a Allure de la contrainte maximale (MPa) en fonction de la profondeur de mesure, $\mathrm{x}(\mathrm{mm})$. l'entaille par le gel est plus élevée dans le cas du Larrys que dans les cas du Chamesson et de la Pierre de Lens. Par ailleurs, alors que pour le Larrys, on observe une forte augmentation du maximum de pression avec la profondeur, ce phénomène est beaucoup moins marqué dans le cas du Chamesson. Pour la Pierre de Lens, on observe même une très légère diminution à partir du milieu de l'entaille.

\section{4}

\section{Rôle du réseau poreux dans la sensibilité à la macrogélivation des calcaires}

\section{4he}

\section{Un processus de gel différent selon le réseau poreux}

Le réseau poreux de la roche module la sollicitation mécanique à laquelle elle est soumise lors du gel de l'eau dans son réseau de fissures.

Si la roche s'avère peu poreuse (comme le Larrys), l'eau contenue clans les quelques gros pores dont la température de congélation est à $0^{\circ} \mathrm{C}$, gèle rapidement. Le palier à $0^{\circ} \mathrm{C}$ est effectivement plus bref avec le Larrys qu'avec des calcaires plus poreux (Chamesson, Pierre de Lens) (Fig. 6). L'eau contenue dans le fond de l'entaille n'a pas le temps de geler. De plus, d'après Letavernier (1984), la perméabilité est une fonction décroissante de la porosité et du rayon moyen d'accès aux pores. Par conséquent, la perméabilité d'une roche peu poreuse est déjà faible initialement et va diminuer rapidement suite à la formation de glace dans le réseau poreux. Les migrations d'eau étant ainsi limitées de l'entaille vers la roche, l'eau encore liquide en fond de fissure se trouve confinée. Avec le développement du front de glace dans l'entaille et l'augmentation volumique induite par le passage à l'état solide de l'eau, la contrainte mesurée en fond d'entaille ne peut qu'augmenter.

Si la roche est poreuse (Chamesson, Pierre de Lens), dans un premier temps, les gros pores gèlent à $0^{\circ} \mathrm{C}$ avec une certaine inertie du fait de la quantité d'eau concernée plus importante que dans le cas d'une roche moins poreuse. Ce délai laisse le temps à l'eau de la partie supérieure de l'entaille, à la pression atmosphérique, de se transformer en couche de glace et à l'eau sous-jacente, encore liquide, contenue dans l'entaille de migrer dans le réseau environnant au fur et à mesure que cette dernière se trouve confinée. Ces mouvements d'eau sont d'autant plus facilités que la porosité et/ou le rayon moyen d'accès aux pores étant forts, la valeur de la perméabilité de la roche est élevée (Letavernier, 1984). Ces pertes d'eau de l'entaille et les possibilités d'extrusion de la glace nouvellement formée ne peuvent que diminuer la valeur de la contrainte générée par l'augmentation de volume lors du gel de l'eau dans le réseau de fissures d'une roche poreuse.

Cette hypothèse sur le processus de macrogélivation est en adéquation avec l'observation, au cours du gel, d'une pression non hydrostatique dans l'eau encore liquide en profondeur dans les fissures des roches à gros pores. 


\section{Une contrainte maximale linéairement liée à la perméabilité}

Ces processus de gel dans une fissure mettent en évidence la forte influence de la perméabilité de la roche sur la contrainte maximale générée. Cette dernière mesurée en fond d'entaille a été comparée à la perméabilité pour les trois calcaires testés. Dans le cas strict de la géométrie choisie et de nos conditions d'essais, la contrainte maximale est une fonction linéairement décroissante du logarithme de la perméabilité (Fig. 9). Or, d'après la formule établie par Letavernier (1984), la perméabilité d'une roche est proportionnelle

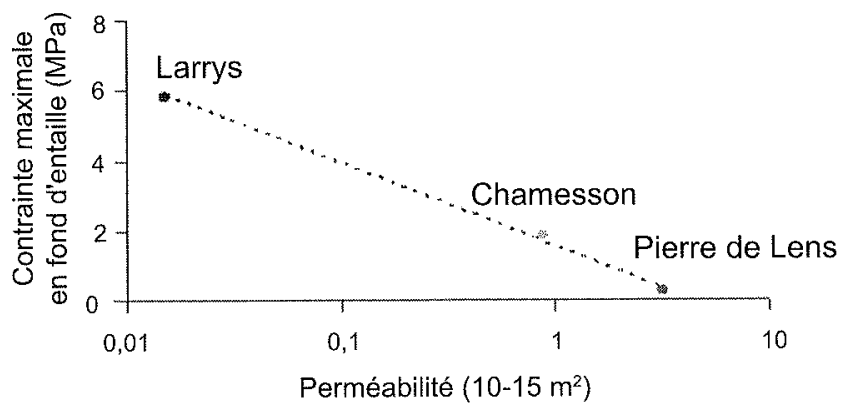

fic. 9 Valeur maximale de la contrainte en fond d'entaille en fonction de la perméabilité pour les trois calcaires testés.

Maximum value of stress at the bottom of the notch versus permeability for the three tested limestones. au produit de sa porosité et du carré de son rayon moyen d'accès aux pores. Par conséquent, la sollicitation générée par le gel dans le réseau de fissures d'une roche calcaire est directement liée aux caractéristiques de son réseau poreux.

Considérant le réseau poreux et la résistance de la roche, la sensibilité à la macrogélivation des roches calcaires peut être connue.

\section{Conclusion}

La contrainte générée par le gel dans le réseau de fissures des roches calcaires est le fruit du conflit entre la cinétique de formation d'un bouchon de glace à l'entrée des fissures, la cinétique d'écoulement des fluides contenus dans les fissures vers le réseau poreux environnant et la cinétique de progression du front de gel dans le réseau poreux de la roche. Dans le cas d'un modèle simple d'une fissure unique, la contrainte maximale générée est proportionnelle au logarithme de la perméabilité. Dans le cas réel d'un réseau de fissures, il faudrait tenir compte de sa tortuosité qui peut avoir son importance sur le confinement créé par la formation progressive de glace. Par ailleurs, l'épaisseur des fissures relativement à la taille des gros pores pourrait avoir son importance dans la primauté de l'une ou l'autre des cinétiques.

Cette étude a été effectuée sur des roches calcaires. Elle doit maintenant être étendue à d'autres types de roches afin de généraliser ces résultats. Elle demande également à être validée par l'étude de cas extrêmes : calcaires de très fortes et très faibles perméabilités.

\section{Bibliographie}

Bost M. - Altération par le gel des massifs rocheux : étude expérimentale et modélisation des mécanismes de génération des contraintes dans les fissures. Thèse, École nationale des Ponts et Chaussées, 2008, $281 \mathrm{p}$

Bousquié P. - Texture et porosité de roches calcaires. Relations avec la perméabilité, l'ascension capillaire, la gélivité, la conductivité thermique. Thèse, université Pierre-et-Marie-Curie, Paris, 1979, 191 p.

Caicedo Hormaza B. - Contribution à l'étude de la migration de l'eau dans les sols pendant le gel et le dégel. Thèse, École centrale, Paris, 1991, 108 p.

Frayssines M. - Contribution à l'évaluation de l'aléa éboulement rocheux (rupture) Thèse, université Joseph-Fourier, Grenoble, 2005, $218 \mathrm{p}$.

Hall K., André M.F. - New insights into rock weathering from high-frequency rock temperature data : an Antarctic study of weathering by thermal stress. Geomorphology 41, 2001, p. 23-25.

Interreg IIC, Groupe Falaise - Prévention des mouvements de versants et des instabilités de falaises. Confrontation des méthodes d'étude des éboulements rocheux dans l'arc alpin. Programme IIC - (c Falaises ») Méditerranée occidentale et Alpes latines, 2001, $239 \mathrm{p}$.

Letavernier G. - La gélivité des roches cal- caires. Relations avec la morphologie du milieu poreux. Thèse, université de Caen, 1984, $181 \mathrm{p}$.

Matsuoka N. - Direct observation of frost wedging in alpine bedrock. Earth Surf. Process. Landforms 26, 2001a, p. 601-614.

Matsuoka N. - Microgelivation versus macrogelivation : toward bridging the gap between laboratory and field frost weathering. Permafrost and Periglacial processes 12, 2001b, p. 299-313.

PIARC G2 - Natural Disaster Reduction for Roads. Final Report, 1999.

Tourenq C. - La gélivité des roches : application aux granulats. Rapport de recherche ${ }^{\circ} 6$, LCPC, Paris, 1970, $60 \mathrm{p}$. 